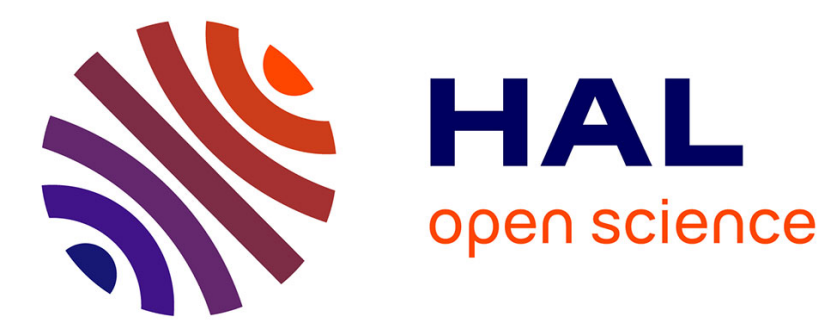

\title{
Auto-management of energy in IoT Networks
}

\author{
Ahmed Zouinkhi, Hayfa Ayadi, Thierry Val, Boussaid Boumedyen, Mohamed
}

Naceur Abdelkrim

\section{To cite this version:}

Ahmed Zouinkhi, Hayfa Ayadi, Thierry Val, Boussaid Boumedyen, Mohamed Naceur Abdelkrim. Auto-management of energy in IoT Networks. International Journal of Communication Systems, 2019, 33 (1), pp.0. 10.1002/dac.4168 . hal-02901798

\section{HAL Id: hal-02901798 \\ https://hal.science/hal-02901798}

Submitted on 17 Jul 2020

HAL is a multi-disciplinary open access archive for the deposit and dissemination of scientific research documents, whether they are published or not. The documents may come from teaching and research institutions in France or abroad, or from public or private research centers.
L'archive ouverte pluridisciplinaire HAL, est destinée au dépôt et à la diffusion de documents scientifiques de niveau recherche, publiés ou non, émanant des établissements d'enseignement et de recherche français ou étrangers, des laboratoires publics ou privés. 


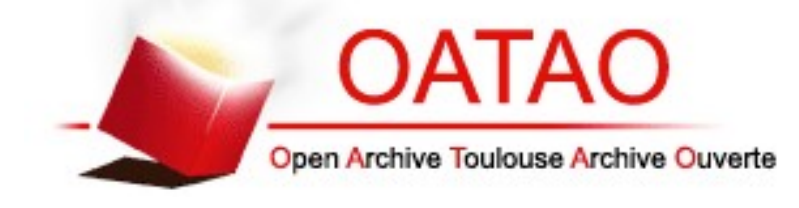

Open Archive Toulouse Archive Ouverte

OATAO is an open access repository that collects the work of Toulouse researchers and makes it freely available over the web where possible

This is an author's version published in: https://oatao.univ-toulouse.fr/26258

Official URL :

https://doi.org/10.1002/dac.4168

To cite this version:

Zouinkhi, Ahmed and Ayadi, Hayfa and Val, Thierry and Boumedyen, Boussaid and Abdelkrim, Mohamed Naceur Automanagement of energy in IoT Networks. (2019) International Journal of Communication Systems, 33 (1). ISSN 1099-1131

Any correspondence concerning this service should be sent to the repository administrator: tech-oatao@listes-diff.inp-toulouse.fr 


\title{
Auto-management of energy in IoT networks
}

\author{
Ahmed Zouinkhi' ${ }^{1}$ | I Hayfa Ayadi ${ }^{1}$ (i) | Thierry Val $^{2}$ | Boumedyen Boussaid ${ }^{1}$ | \\ Mohamed Naceur Abdelkrim ${ }^{1}$
}

${ }^{1}$ Research Laboratory Modeling, Analysis and Control of Systems (MACS), University of Gabes, Gabes, Tunisia

${ }^{2}$ Institute of Research in Computer Science of Toulouse, University of Toulouse, Toulouse, France

\section{Correspondence}

Ahmed Zouinkhi, Research Laboratory Modeling, Analysis and Control of Systems (MACS), University of Gabes, Gabes, Tunisia.

Email: ahmed.zouinkhi@enig.rnu.tn

\begin{abstract}
Summary
Wireless sensor network (WSN) based on IEEE 802.15.4 technology continues to gain considerable importance. The present work is based on this standard and a cluster tree network topology. This network is divided into many subgroups, each of which is characterized by its special IEEE parameters (beacon order [BO] and superframe order [SO]). A mathematical model for computing energy consumption was proposed to enable personal area network (PAN) coordinator to detect the current energy level in the battery. The proposed adaptive intervention is based on a comparison between the remaining energy and the diverse thresholds, which lead to an auto-management of energy consumption. The proposed approach was evaluated using Inetmanet/Omnet++ simulator.
\end{abstract}

\section{KEYWORDS}

auto-management, energy, IEEE 802.15.4, IoT, WSN

\section{I | INTRODUCTION}

Nowadays, the Internet of Things (IoT) is integrated in all fields of technology. It is known as the Industrial Internet or Internet of Everything. IoT has recently been implemented to maintain the connectivity of various devices without any human intervention. The term "Things" includes all kinds of machines, devices, and actuators designed to be used in the social life of human beings. ${ }^{1}$

IoT has gained much interest thanks to its various advantages. It is considered as an extension of machine-to-machine communication (M2M) in order to maintain the communicative interaction between everyone and everything. All kinds of "things" are enabled to be connected with each other. Many tasks could be performed by these things such as detecting data and sensing it wirelessly, which reflects their intelligence. These smart communicating things have the capacity to evaluate the data collected from the sensing materials in order to take the most appropriate decision about them. It allows all objects to communicate with each other to be able to adapt to their environment by sensing some physical parameters.

The IoT is implemented in different kinds of applications: smart home, ${ }^{2}$ transportation, ${ }^{3}$ agriculture, ${ }^{4}$ health care, ${ }^{5}$ industry, ${ }^{6}$ and entertainment and surveillance ${ }^{7}$ (Figure 1).

Despite the different advantages of IoT systems, they also suffer from many problems such as the number of connected devices that request a private address (identification) in addition to the necessity of having a dynamic system that could take into consideration the immense quantity of nomenclature of the different devices. ${ }^{8}$ Moreover, IoT systems must be able to associate a unique and specific identity for each object. Because of the big diversity of the connected objects, many problems could arise, such as the interoperability and standardization, which present the most serious issues. As it is a kind of data transmission, an encryption system always remains an important issue in saving the data transmitted. Faults detection $^{9,10}$ and energy consumption present well-known troubles behind the development of new techniques aiming to manage the energy consumed by wireless sensor networks (WSNs) and IoT systems properly. ${ }^{11,12}$ Indeed, as the data 
FIGURE 1 Internet of Things applications

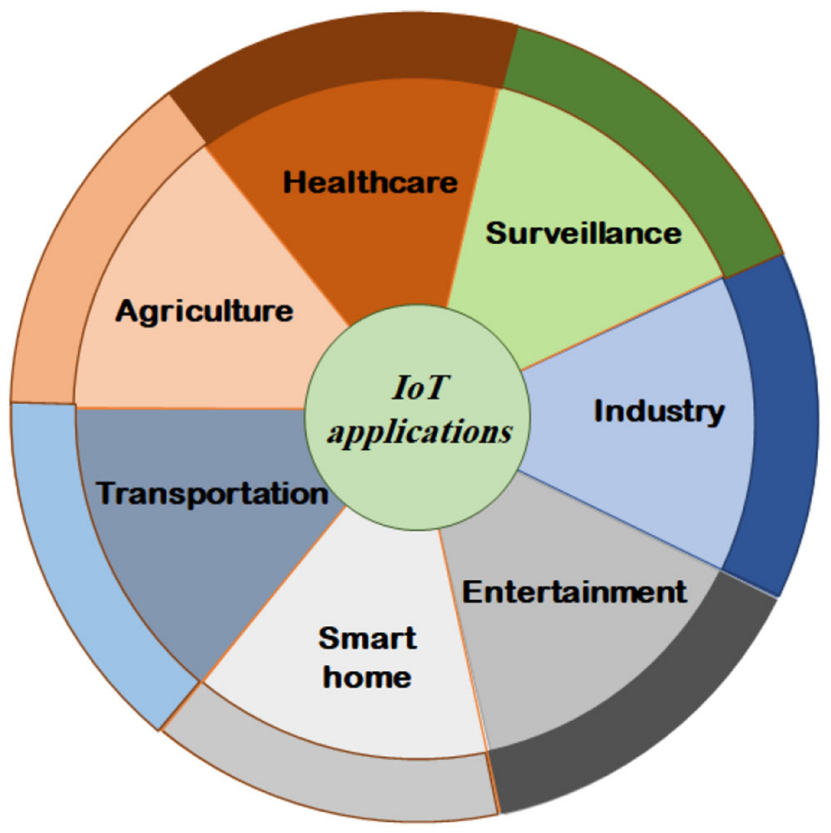

rate grows, the consumed energy continues to increase remarkably. Another challenge facing the IoT systems lies in the limited spectrum available, which leads to the implementation of a dynamic cognitive spectrum allocation technique with respect to the entirety of implicated devices ${ }^{10}$.

So, it is crucial to control the protocols used in each level and each phase over the deployment of simulators or test bed. In addition, the IoT systems are about connecting billions, or trillions, of different objects via the Internet network. Various models were invented in order to propose a clear architecture for the IoT systems, but all of them suffer from several shortcomings, such as the quality of service (QoS), reliability, scalability, and interoperability.

Energy consumption remains the most critical challenge, which leads to developing many new strategies such as routing protocols. ${ }^{13-19}$ The fusion technique also presents an interesting strategy to solve this problem. ${ }^{20,21}$ Several other methods have been established to reach the same goal, such as the anti-collision algorithm, ${ }^{22}$ which consists of mapping correlation of identification (ID) for radio frequency identification (RFID). This method can increase the association between tags so that they can send their own ID under certain trigger conditions by mapped ID correlation. Game theory ${ }^{23}$ and learning methods ${ }^{24}$ are thus used for solving energy issue. Even in mobile networks, this trouble was treated extensively by researchers, for example, in previous studies. ${ }^{25-30}$

In Zhang et al, ${ }^{31}$ the authors developed a new approach that is based on reducing the noise problem in the network by using spherical coordinates systems. A new access protocol was proposed in Zhang et al, ${ }^{32}$ aiming to increase data reliability. Reducing energy consumption leads in many times to change in many network parameters, namely, the network topology ${ }^{33,34} \mathrm{An}$ interesting method was proposed in Zhang et $\mathrm{al},{ }^{35}$ which deals with the creation of new agents. Moreover, in order to reach the same objective, many sensing and aggregation methods were developed, for instance, in Ma et $\mathrm{al}^{36}$ and Zhang et al. ${ }^{37}$ The energy problem has been the subject of several studies in all kinds of networks, such as ad hoc networks, ${ }^{38}$ MANET network, ${ }^{39}$ and mobile network..$^{40,41}$

The satellite network in navigation satellite system, which is necessary for mobile monitoring, has many disadvantages such as asymmetric bandwidth, unstable network, and high bit error rate. This is a new challenge to the existing congestion control method. In the present work, in order to improve the performance of the congestion control mechanism, we suggest TCP-QS (quick start) from the TCP protocol control, which mainly optimizes the slow start stage.

The main interest of the present paper is energy management in the IEEE 802.15.4 technology. The personal area network (PAN) coordinator detects the energy level in the batteries of the nodes. Many thresholds are set. In the case when the remaining energy is inferior to the threshold, the PAN coordinator intervenes by changing the two IEEE 802.15.4 parameters (the beacon order [BO] and superframe order [SO]) in order to increase the lifetime of the node.

The rest of paper is organized as follows: Section 1 describes the different applications of WSN enabled by IoT systems. A general overview of the most well-known works dealing with the IEEE 802.15.4 is presented in Section 2. Section 3 presents the major technologies used in the IoT systems such as the Z-Wave, low-power wireless PAN (6LoWPAN), ZigBee, 
and the IEEE 802.15.4. Section 4 is devoted to the explanation of the proposed approach. The simulation results in diverse states of data transmissions are presented in Section 6. The paper ends with the major conclusion and the most important future works.

\section{I RELATED WORK}

Energy consumption presents one of the most pertinent problems in WSN, which is why it has been the subject of many research works, such as Zhang et al. ${ }^{42-44}$ With the IEEE 802.15.4 technology, there are three possible approaches concerning the consumed energy by each node. They suggest intervening with the superframe duration (SD), intervening with beacon interval (BI), or adjusting both parameter values (SD and BI). In fact, reducing the active period is the most useful strategy and the simplest alternative. Much research has been conducted to prove these strategies. ${ }^{45}$ A Markov model was developed in Choudhury et $\mathrm{a}^{46}$ to study the IEEE 802.15.4, especially the carrier sense multiple access with collision avoidance (CSMA/CA). They estimate the delay and energy consumption all along the transmission of frames using medium access control (MAC) parameters. They proposed a different approach, named dynamic superframe adjustment algorithm (DSAA), in which the SO value can be selected on the basis of a small comparison, to be established in terms of the certain network associated parameters, such as the collision rate. ${ }^{47}$ In turn, the authors have introduced another approach, which they called the duty cycle algorithm (DCA), in which they considered adjusting the SO parameter with reference to the queuing delay, the queue size, the data rate, and the energy consumption. To this end, they used a fixed value of $\mathrm{BO}$ and a variable $\mathrm{SO}$ value, which leads to conserving the $\mathrm{BI}$ that affects the sleeping period. Additionally, the adaptive algorithm to optimize the dynamics (AAOD) also stands as another useful model for dealing with the SO value management. An interesting technique was proposed in Latif et al. ${ }^{48}$ The authors describe a traffic-aware dynamic superframe adjustment (TDSA) algorithm, which manages the superframe via the two MAC layer parameters: BO and SO. The duty cycle formed by these two parameters is adapted on the basis of the number of source nodes, the application data rate, and the received ratio.

The adaptation of the SO parameter depends on the number of data packets received. In the case where their number exceeds a predefined threshold, the SO value would then be changed, although in the opposite case, the SO would increase. However, another protocol named the adaptive MAC protocol (AMPE), highly dependent on the superframe occupied period, is also considered to fit well with this very interesting technique. ${ }^{49}$ The smart duty cycle adaptation (SDCA) algorithm was proposed in Latif et $\mathrm{al} .{ }^{48}$ The authors adopted the duty cycle of nodes simultaneously. This algorithm adapts the duty cycle in real time on the basis of network load, network delay, and received ratio. Moreover, by establishing a small comparison with the first threshold, superframe occupation ratio turns out to be inferior to the initial threshold and the next test needs to be administered, whereby the occupation ratio would be compared with a second threshold.

Indeed, an individual beacon order adaptation (IBOA) algorithm for IEEE 802.15.4 networks was proposed in Gao and $\mathrm{He}^{50}$ in which the node suffering from energy problems could be adjusted. The BI and the duty cycle of each node are adapted in order to improve the performances of power consumption and end-to-end delay. In Oliveira et al, ${ }^{51}$ the authors adjust the duty cycle by intervening simultaneously with the BI and SD values. The duty cycle self-adaptation algorithm (DBSAA) presents the most efficient technique. Thus, the duty cycle depends on four network parameters, which are the number of source nodes, the number of packets received by the coordinator, the collision ratio (CR), and the superframe occupation ratio (OR).

\section{3 | IOT SYSTEM'S PERTAINING TECHNOLOGIES}

The IoT network consists of a joint interaction among a wide range of various technologies, involving a large array of sensors, culminating in the emergence of the WSN. In fact, the WSN is considered as the major contributor in IoT, in which a great number of sensors are intricately interconnected, and inter-nodal data turn out to be transmitted in a way that gives birth to networks of smaller range. Many technologies could be used such as the ZigBee and the IEEE 802.165.4 in addition to other protocols like the Z-Wave and 6LoWPAN. ${ }^{52}$

\section{1 | Z-Wave}

The Z-Wave enables data transmission between the PAN coordinator and all the nodes in the range of its relevant transmission area. It consists of five major layers, namely, the MAC, physical, routing, transfer, and application layers considering the $868 \mathrm{MHz}$ in Europe and $908 \mathrm{MHz}$ in the US. The data rate could reach even 20kbps. Regarding the MAC layer, it is 
designed to establish the collision avoidance scheme. The nodes are classified into two classes, which are the controllers group and the slave nodes.

\subsection{Low-power wireless personal area network}

The 6LoWPAN protocol maintains the collaboration between the IPv6 and the low-power wireless area networks..$^{52}$ The 6LoWPAN is based on low-cost devices implemented in the IEEE 802.15.4 with both its physical and MAC layers in addition to other layers, such as the transport layer, the application layer, the adaptation layer, and the network layer. The 6LoWPAN is designed as the most pertinent platform, perfectly adapting to transmit the IPv6 via IEEE 802.15.4. The IPv6 is invented with the sixth development of the IP address for the network communication, which leads to the development of the 6LoWPAN as a substitute to the 4LoWPAN. As with all other protocols, the 6LoWPAN presents some limitations such as the transmitted packets' size and a number of shortcomings, such as the considerably low bandwidth, power-shortage troubles, and connectivity problems, along with the devices' capacity volume.

\section{3 | ZigBee}

The ZigBee is considered as the most convenient technology in spite of the great number of connected technologies. It is defined as the most useful in applications with low-rate sensors. It is formed by the physical layer in addition to the MAC layer, the application layer, and the network layer. As with the 6LoWPAN, the ZigBee is based on the IEEE 802.15 especially in both physical layer and MAC layer. Although the upper layer is defined by its proper technology, it enables two kinds of devices, which are the full function device (FFD) and the reduced function device (RFD) that allow three different kinds of topologies: the mesh, the tree, and the star topologies. Moreover, it is a typical classification of nodes into three kinds of nodes, the router, the end devices, and the coordinator. The coordinator is responsible for the choice of the most suitable route in order to maintain the data transmission and the most appropriate topology used for keeping the efficient functioning of the network and for loading all the other parameters, including the channel's frequency and the network's identifier, and for maintaining the operational parameters. ${ }^{52}$ The end device is characterized by its low power consumption. As for the end device, it is characterized by a low-rate and low-power capacity, involving a number of environmental parameters' detecting devices. Along with the third components, the routers form the most important instruments because of their responsibility to ensure the coordination of activities between both the end devices and the coordinator nodes. It can keep an easy connection between the routers of the network.

\section{4 | IEEE 802.15.4}

The IEEE 802.15.4 is the principal technology in the IoT networks. ${ }^{53}$ It was invented to solve similar challenges facing the WSN such as the low-rate, versus the great area, coverage associated with the WMAN and WLAN networks. The energy consumption remains the major problem confronted by most of the wireless communication, including the WPAN family, which is constituted by just physical and MAC layers. As with the ZigBee technology, its associated nodes could be of an FFD or an RFD type. The main related imposition is that the coordinator node must obligatorily be an FFD node, although the end-device could be an RFD in type. Indeed, the FFD nodes are remarkable with their remarkable energy capacity compared with the RFD nodes. The topological models enabled to fit well for an interactive co-integration with this particular technology are the star and the peer to peer, in addition to mesh architectures. With respect to the star topology, the communication must be ensured between one PAN coordinator and at least a single end device. In the peer-to-peer topology, the data transmission could be ensured between a couple of nodes of the same type.$^{53}$ In mesh architecture, each node is apt to transmit its data to any other node in the network. The IEEE 802.15.4 technology encloses four major layers: the MAC layer, the physical layer, the network layer, and the application layer. The physical layer is responsible for keeping the data services required by managing the 27 channels for the three bands available, specifically, 10 channels within the $915 \mathrm{MHz}$ range and a single channel within the $868 \mathrm{MHz}$ range, along with 17 channels within the $2.4 \mathrm{GHz}$ range band. Three data rates could be set by the IEEE 802.15.4, which are 20, 40, and $250 \mathrm{kbps} .{ }^{53}$ The MAC layer undertakes the most interesting functions, namely, ensuring the link quality indication (LQI), monitoring and dealing with the radio transceiver's activity, managing the beacon relevant data, and selecting the appropriate transmission channel in addition to energy detection (ED). Moreover, the MAC layer is the best manager of the beacon transmission process in addition to the guaranteed time slot (GTS), achieving the crucial role of ensuring data transmission security. ${ }^{53}$

In this respect, the IEEE 802.15.4 technology helps in providing two modes of activity: the beacon-enabled mode and the non-beacon-enabled mode. Regarding the former, the PAN coordinator periodically sends a beacon frame to the entirety of the network's nodes to maintain a full range synchronization of their activities in addition to controlling the nodes' 
duty cycle through determining their relevant activity periods and sleeping spans, as defined via both the SD and the BI, ${ }^{54}$ which are described by relations (1) and (2), respectively.

$$
\begin{gathered}
B I=\text { aBaseSuper frameDuration } * 2^{B O} ; \\
1 \leq \mathrm{B} \leq 14, \\
S D=\text { ABaseSuper frameDuration } * 2^{S O} ; \\
0 \leq \mathrm{SO} \leq \mathrm{BO} \leq 14 .
\end{gathered}
$$

The SD duration specifies the activity duration of nodes in which the node is able to communicate with other nodes in the network. Indeed, adjusting the SD duration would certainly contribute to controlling the energy consumed by the node. As for the second duration interval, it concerns the situation in which the node still remains in a sleeping state. ${ }^{53}$ The SD duration is equal to 16 equal-sized slots (Figure 2). The frame of the IEEE 802.15.4 is formed by three parts, which are the beacon frame, the contention access period (CAP), and the contention-free period (CFP). During the CAP, the node has the choice of sending data via the CSMA/CA protocol. However, in the CFP interval, the time division multiple access (TDMA) protocol is used. There are two possible modes for the CSMA/CA protocol, namely, slotted and unslotted versions. The present work is interested in the slotted mode of the CSMA/CA as presented in Figure $3 .{ }^{54}$

In the first level, we start by defining the relevant parameters, which are $C W$, which describes the congestion window, with $(N B)$ presenting the number of times the slotted algorithm proves to request the congestion window back-off, and $B E$, which represents the back-off exponent. In the second level, two clear channels are awaiting to explore the transmission

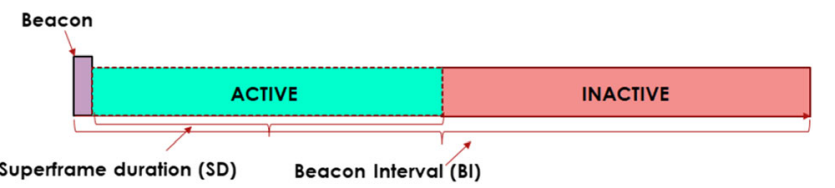

FIGURE 2 Frame composition

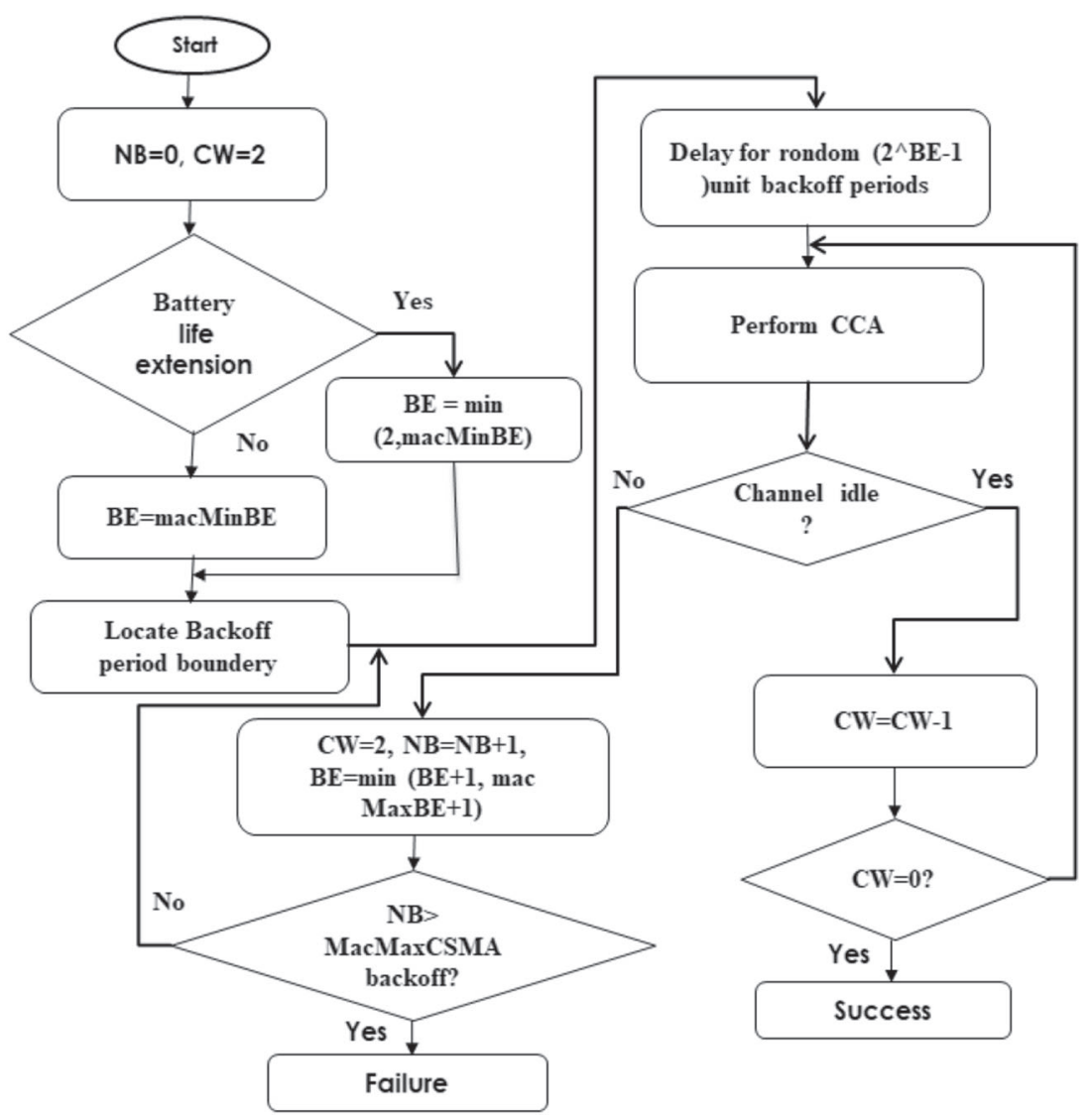

FIGURE 3 Carrier sense multiple access with collision avoidance (CSMA/CA) slotted algorithm 
channel associated potential. Afterwards, on the basis of the canal status, a relevant appropriate decision is taken. Then, either relevant data will be transmitted or an extra short duration is awaited.

\section{I THE PROPOSED APPROACH}

The present work aims to properly adjust the last quantity of energy remaining in a node's battery. It consists of monitoring the two IEEE 802.15.4 beacon-enabled mode (BO, SO) values. The present methodology is applied to a highly extended network based on cluster tree topology.

The first step consists of collecting the data from all the nodes of the network. Therefore, every node transmits its data to its corresponding coordinator, which computes its battery level $E_{R}$. Then, $E_{R}$ is compared with the initial energy threshold of $E t 1$. In the case where the $E_{R}$ is less than $E t 1$, the $\left(B O_{1}, S O_{1}\right)$ are then computed by the corresponding coordinator.

The same procedure is repeated with all nodes and thresholds. The four levels treated by our approach are L1, L2, L3, and L4, which are equal to 250,200,150, and $100 \mathrm{~J}$ (Figure 4). The different steps of the proposed approach are illustrated in Figure 5.

FIGURE 4 Energy levels
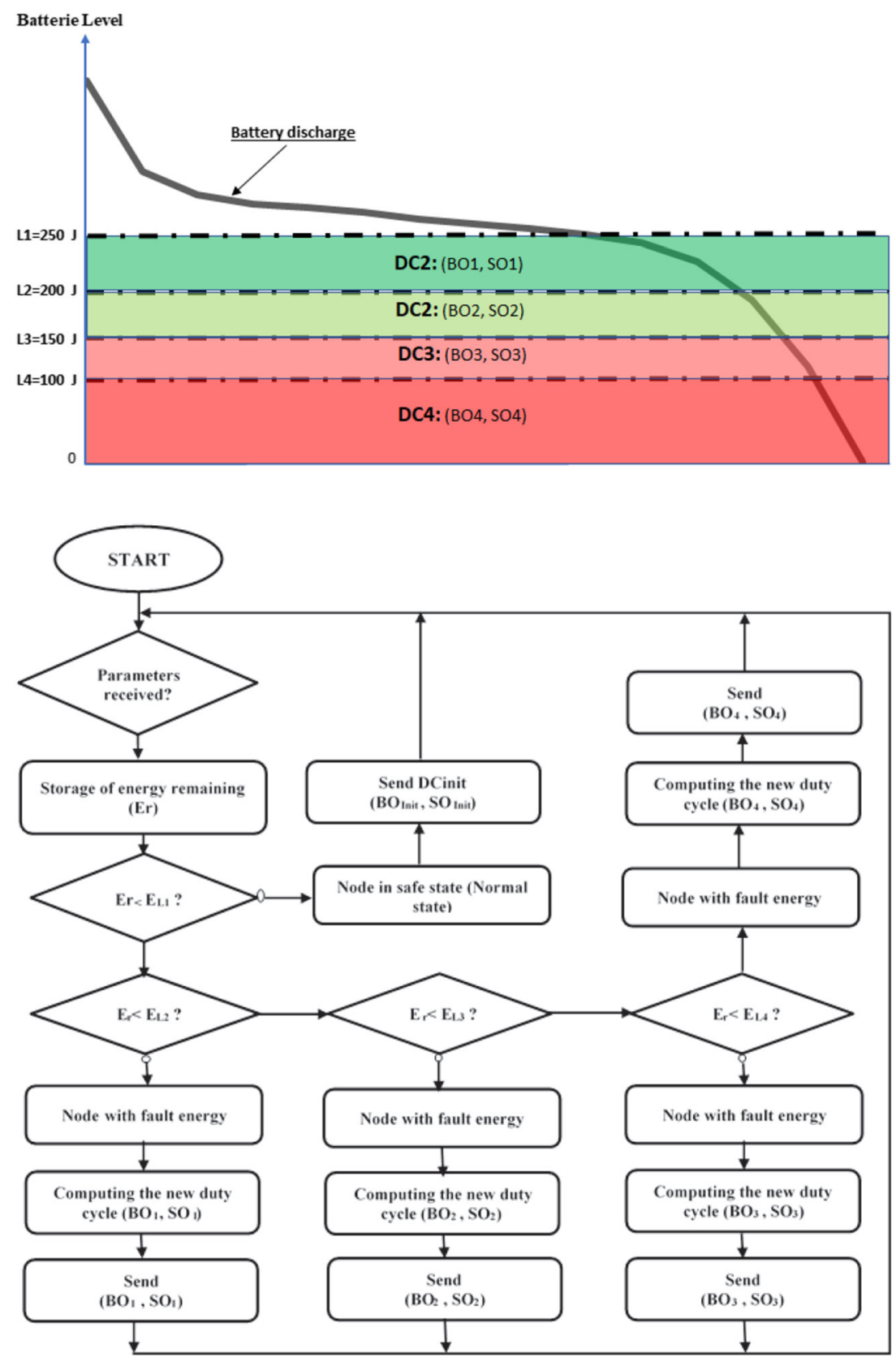


\subsection{Energy formula}

The energy wasted in transmission states could be considered as the quantity wasted by every byte sent during the SD period in addition to the quantity wasted when the antenna is in transmitting state. During the transmission period, the node consumes the most important quantity of energy, named $E_{\text {em }}$, which is described by the equation below:

$$
E_{\text {em }}=\left(n b t_{s d} * T t t * E b\right)+2 * U * I * C C A * T p_{b a c k}
$$

$n b t s d$ presents the number of data frames forming the SD; $I$ is the current value; $U$ presents the voltage value; $E b$ is the binary energy; $\mathrm{Ttt}$ describes the frame size; $C C A$ is the clear channel assessment; and $T p_{b a c k}$ is shown by formula below:

$$
T p_{b a c k}=\left(2^{\text {cst }} t_{\text {back }}-1\right) * 20 \text { symbol. }
$$

The cst $_{\text {back }}$ is for the backoff period. Indeed, the node loses some quantity of energy even in receiving information, named $E_{r c}$. It is all the quantity of energy waste in receiving every byte in the SD duration described by Equation (5):

$$
E_{r c}=n b r_{S D} * E b
$$

$n b r_{S D}$ is the number of bits used in the SD duration. In addition, even during both of the overhearing and overmitting phases, some quantity of energy is consumed, as described by $E_{0}$.

The overhearing is the phase in which the node hears data that are not sent for it. The overmitting is the state in which the node sends data when its receiver node is not ready to receive it yet. In order to compute the quantity of energy wasted in it, we use the error rate PER, which reflects the amount of data wasted in the network because of the overhearing or the overmitting problem as presented by formula (6).

$$
E_{o}=n b r_{t r} * d t r a * E b * P E R .
$$

$d t r a$ describes the size of the frame (the bit); $n b r_{t r}$ presents the number of bits sent by the node; $E b$ presents the binary energy; and PER is the error rate reached when the packet is transmitted without being well received. In order to detect the energy consumed in the collision state, we resort to the period needed to access the transmission canal Tatt, which reflects the state of message collision in the collision canal. So, we compute $\mathrm{Nbpk}$, which is the number of attempts that the node has made in order to transmit data without receiving any acknowledgment in addition to the current value and the voltage value during this period. The energy consumed in collision period $E_{c o l}$ is via formula (7):

$$
E_{c o l}=\text { Tatt } * U * I * N b p k
$$

Indeed, the sleep technique is the best technique used to control the energy consumed in order to manage the lifetime of the node. The energy consumed throughout the sleep period $E_{S L P}$ is expressed via the equation below. It is gotten via detecting the sleep period (BI-SD) and the binary energy.

$$
E_{S L P}=E b *(B I-S D),
$$

although the energy consumed during the idle state could be calculated with equation (9). The idle state is defined as a short inter-frame spacing in which the node waits for the acknowledgment from the receiver node. To obtain the quantity of energy, we use all the period of time $T_{S} I F S$ and the quantity of power and current spent in this time.

$$
E_{\text {idle }}=T_{S} I F S * U * I .
$$

The aim of this work is to manage the lifetime of the nodes, which are organized with cluster tree topology and manipulated with the IEEE 802.15.4 beacon-enhanced mode. The network is formed by many subgroups, each one each of which is formed by a PAN coordinator and a number of nodes and which is characterized by special parameters of (BO, SO) of the IEEE 802.15.4. 
Each node sends its specific parameters to its PAN coordinator periodically, which enables the last one to compute its current energy level and its remaining energy $E_{R}$. In the case where the value of the remaining energy is less than the threshold, the coordinator executes the proposed technique in order to get the most appropriate value of (BO, SO). The same algorithm is repeated four times with all the thresholds set. Indeed, the quantity of energy remaining in the battery is computed as the difference between the initial energy Eini and the consumed energy Ec. It is expressed via Equation (10):

$$
E_{R}=\text { Eini }-E c .
$$

It can be obtained as the sum of all the energy consumed in different modes as presented by Equation (11):

$$
E c=E_{e m}+E_{c o l}+E_{o}+E_{S L P}+E_{r c}+E_{\text {idle }}
$$

\subsection{The IEEE 802.15.4 parameter's intervention}

Our intervention is manifested on many steps. The first stage is about collecting the serious parameters from all the nodes of the network in order to get the consumed energy $E C$. In a second stage, the $E C$ is compared with a specified threshold. Indeed, in the beacon-enabled duration, the node-consumed energy turns out to be provided by formula (12). It is defined as the number of frames existing in the $B I$ interval multiplied by the energy consumed by a single frame $E_{T}$.

$$
\mathrm{E}_{\mathrm{BI}}=\frac{\mathrm{BI}}{T_{t \mathrm{t}}} * \mathrm{E}_{T}
$$

$T_{t t}$ is the length of the frame, and BI presents the BI period. The BI lost energy is assumed to be less than the energy consumed, as described by expression (13):

$$
E_{B I} \leq E_{R}
$$

Both relations (12) and (13) give birth to formula (14):

$$
\frac{B I}{T_{t t}} * E_{T} \leq E_{R}
$$

The BI interval is set by the IEEE 802.15.4 standard as expressed through formula (15):

$$
B I=15,36 * 10^{(-3)} * 2^{B O}
$$

So, expression (14) is changed into relation (16):

$$
\left(15,36 * 10^{(-3)}\right) * \frac{2^{B O}}{T_{t t}} * E_{T} \leq E_{R}
$$

On the basis of equation (14), $2^{B O}$ is defined by Equation (17):

$$
2^{B O} \leq \frac{E_{R} * T_{t t}}{15,36 * 10^{(-3)} * E_{T}} .
$$

By adopting a logarithmic passage, the $B O$ value is presented by relation (18).

$$
B O * \log (2) \leq \frac{E_{R} * T_{t t}}{15,36 * 10^{(-3)} * E_{T}}
$$

On the basis of a major assumption, the $B O$ value is presented by relation (19):

$$
B O=\frac{\log \left(\frac{E_{R} * T_{t t}}{15,36 * 10^{(-3)} * E_{T}}\right)}{\log (2)} .
$$




\begin{tabular}{|ll|}
\hline Parameters & Values \\
\hline Simulation time & $100 \mathrm{~s}$ \\
\hline Network size & $(800 \mathrm{~m}, 400 \mathrm{~m})$ \\
\hline Initial energy & $18720 \mathrm{~J}$ \\
\hline Nodes number & 24 \\
\hline PAN number & 8 \\
\hline Sink number & 1 \\
\hline Channel frequency & $2.4 \mathrm{GHz}$ \\
\hline Radio type & IEEE 802.15 .4 \\
\hline
\end{tabular}

TABLE 1 Simulation parameters

Abbreviation: PAN, personal area network.

For a good management of the remaining energy in the battery, just $10 \%$ of the remaining power is employed, named $E_{R 1}$, as presented by expression (20):

$$
E_{R 1}=E_{R} * 0.1
$$

Therefore, $B O$ is expressed by relation $(21)^{11}$ :

$$
B O=\frac{\log \left(\frac{0.1 * E_{R} * T_{t t}}{15,36 * 10^{(-3)} * E_{T}}\right)}{\log (2)} .
$$

The superframe duration $S D$ represents just $70 \%$ of the $B I$ duration. Thus, the $S O$ value is expressed through Equation (22):

$$
S O=0.7 * \frac{\log \left(\frac{0.1 * E_{R} * T_{t t}}{15,36 * 10^{(-3)} * E_{T}}\right)}{\log (2)} .
$$

\section{I SIMULATION RESULTS}

The simulator Omnet++ is used in our work in order to simulate the proposed approach thanks to its different advantages. It is an objective modular network testbed in $\mathrm{C}++$, and it is designed as the most realistic simulator for WSNs. It is an object-oriented modular and a discrete event network-simulation framework. The OMNET ++ present a multidisciplinary tool manipulated for many objectives. It models both modes of wired and wireless communication. ${ }^{55}$ The INETMANET module is used in our case. The simulation parameters are presented in Table 1.

Our network is formed by 24 nodes, eight PAN coordinators, and a node sink. It is formed by eight subgroups, each of which is composed by a single coordinator and three nodes as illustrated in Figure 6. Every subgroup is characterized by specific IEEE 802.15.4 parameters. The simulation starts by transmitting a beacon frame to the PAN coordinator correspondent. Then, every node sends periodically their interesting parameters to its correspondent PAN that is able to detect its current state.

Every subgroup is characterized by specific IEEE 802.15.4 parameters. The simulation starts by transmitting a beacon frame to the PAN corresponding coordinator. Then, every node periodically sends their interesting parameters to its corresponding PAN that is able to detect its current state. Indeed, the PAN coordinators, named PAN 1, PAN 2, PAN 3, PAN 4, PAN 5, PAN 6, PAN 7, and PAN 8, are mainly responsible for the nodes of the subgroups. Each subgroup has its proper specific BI- and SD-associated parameters, which contribute to the appearance of eight different functional modes in the same network. The variety of functional modes in the same network presents the most interesting and particular aspects of this work. Moreover, the presence of four thresholds $(D C 1, D C 2, D C 3$, and $D C 4)$ leads to the creation of four different modes. Figure 7 presents the different thresholds activated.

When the threshold is reached, the PAN coordinator intervenes with a different value of duty cycle, which depends on new rates of IEEE 802.15.4 parameters (BO, SO) as presented in Table 2. When the PAN node detects that its node suffers from a serious state because its remaining energy is inferior to the set threshold, it intervenes by changing both (BO, SO) values.

This procedure is repeated with the four thresholds. In our case, the node (Pr) is the node that suffers from a serious energy state because the energy remaining in the battery is inferior to the first threshold (L1), which leads to start our algorithm in 100 packets per second of traffic load. The same process is repeated with 300 packets per second when the energy consumed in this node decreases under threshold number 2 (L2), which presents the second intervention and the 
FIGURE 6 Network with multimode topology
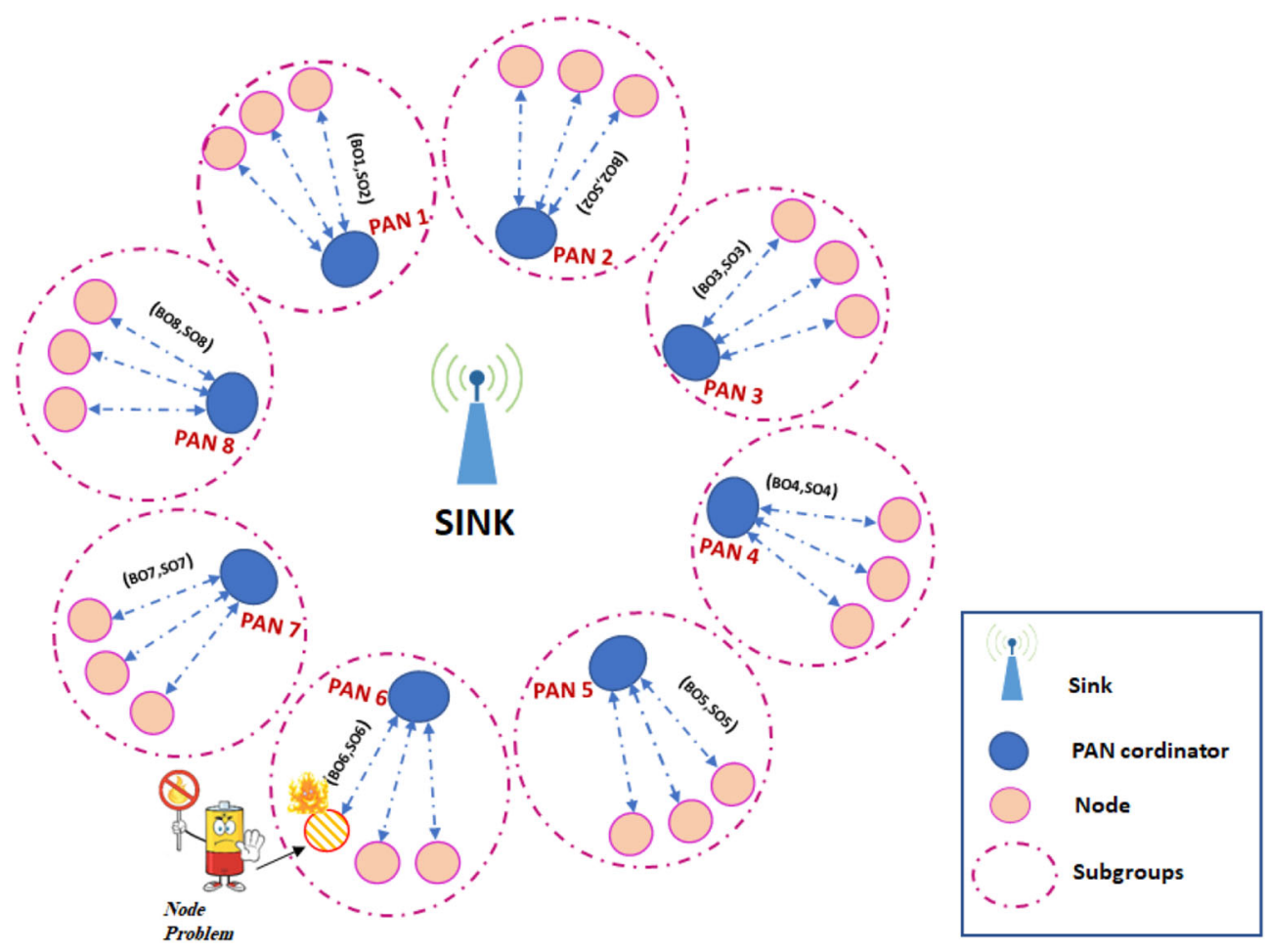

FIGURE 7 Duty cycle change

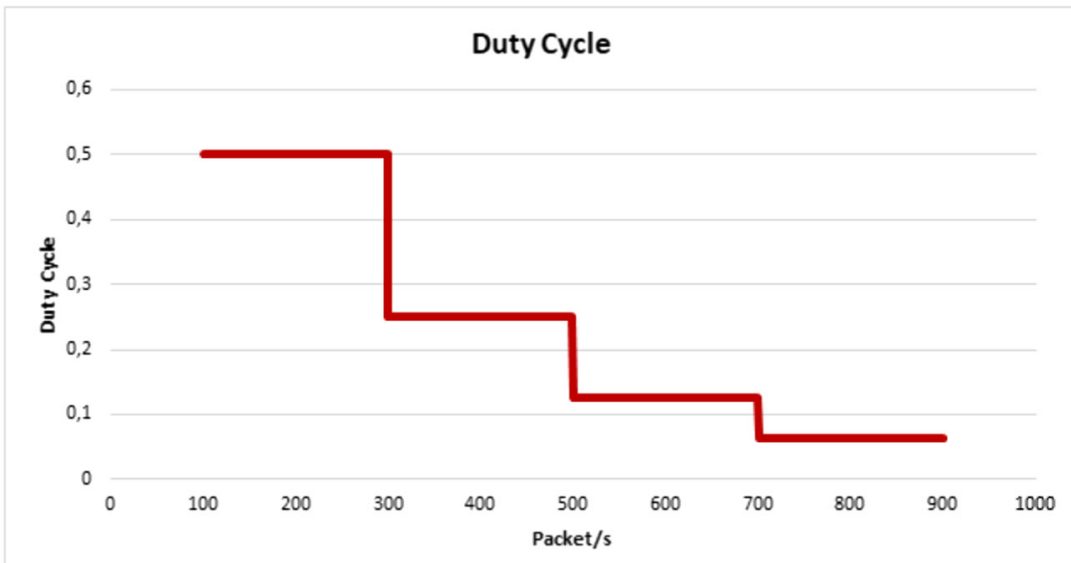

TABLE 2 Configuration of parameters' auto-management

\begin{tabular}{|llllrc|}
\hline Thresholds (J) & DC & BO & SO & BI (ms) & \multicolumn{1}{c|}{ SD (ms) } \\
\hline 250 & $50 \%$ & 3 & 2 & 122.88 & 61.44 \\
200 & $25 \%$ & 5 & 3 & 491.52 & 122.88 \\
150 & $12.5 \%$ & 8 & 5 & 3932.16 & 491.52 \\
100 & $6.25 \%$ & 10 & 6 & 15728.64 & 983.04 \\
\hline
\end{tabular}

Abbreviations: BI, beacon interval; BO, beacon order; DC, duty cycle; SD, superframe duration; SO, superframe order.

second duty cycle (DC2). In addition, at 500 packets per second, the $E C$ continues to decrease until reaching less than L3, which contributes to our third intervention. The last intervention has occurred at 700 packets per second when the remaining energy becomes inferior to threshold number 4 (L4), which presents the fourth duty cycle $D C 4$.

The proposed approach is manipulated by the node $(\operatorname{Pr})$. The presented results are all the performances of this node after each intervention. We compare our results with five other approaches, which are battery-aware beacon-enabled IEEE 802.15.4: the adaptive and cross-layer approach (BARBEI), ${ }^{56}$ the dynamic adaptation of duty cycling (DADC), ${ }^{46}$ the smart Duty Cycle Adaptation (SDCA) ${ }^{48}$ the TDSA, ${ }^{50}$ and the IEEE 802.15.4 with $(\mathrm{BO}, \mathrm{SO})=(7,5) .{ }^{57}$ 

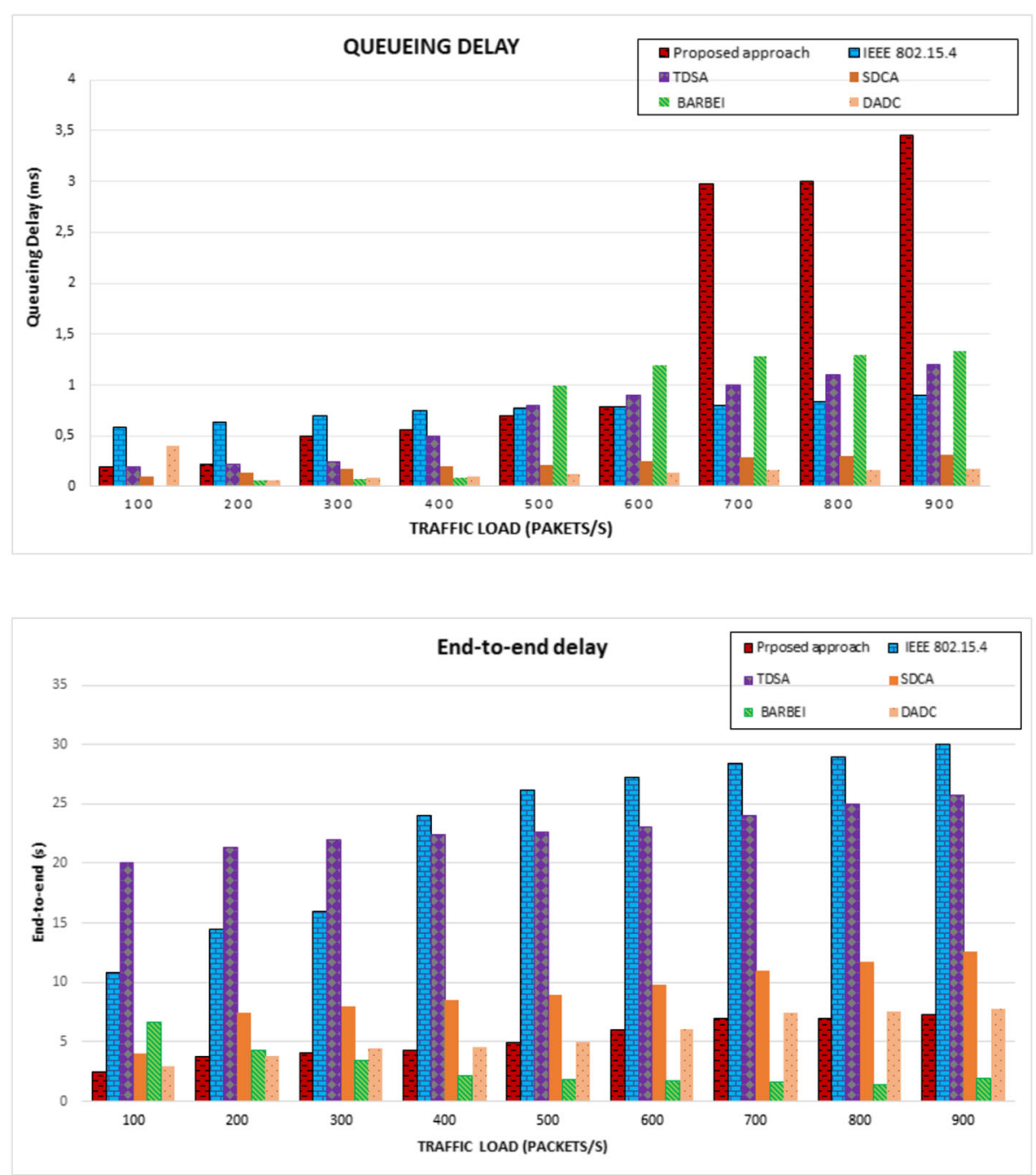

FIGURE 8 Queue delay of the node with fault energy (node number 30)

FIGURE 9 End-to-end delay of the node with fault energy (node number 30)

Many parameters were tested and presented in order to prove the efficiency of the proposed approach, namely, end-to-end duration and the queuing delay, in addition to all modes of energy consumption, namely, states of reception, emission, sleep, and collision with the overhearing and overmitting periods. The traffic load varied from 100 to 900 packets per second.

The queue delay evolution is illustrated by Figure 8. The lowest values of the queue delay are presented by the DADC method when the traffic load is more than 200 packets per second, although the BARBEI approach presents a very important increase after 500 packets per second. For the IEEE 802.15.4, the values of the delay evolution increase slowly from 0.6 to 0.9 second. For the case of the SDCA, the values of the studied approach are so low. It increases by the growth of the packets loads' values. The proposed approach presents very important values, especially with high packet loads.

All the simulated approaches present an increase in the values of the end-to-end delay. In the case of the IEEE 802.15.4, these values of duration increase from 11 to 30 seconds. However, they increase from 20 to 26 seconds in the TDSA approach. For the SDCA, the values increase from 4 to 14 seconds, but the values of the DADC do not reach 10 seconds all over the simulation. The best results are achieved by the method of the BARBEI (Figure 9). The proposed approach attained better results compared with the SDCA, IEEE 802.15.4, and TDSA techniques.

\section{1 | The stable state-related energy consumption}

The node transmission states present two modes, which are stable state and transient state. During the former, the node is busy sending and receiving data. Many states are operated in the first mode such as the overhearing-overmitting, sleeping, receiving, emission, collision, and idle. Figure 10 presents the simulation results of the collision state in which the consumed energy increases with the growth of data packets at very important speed, especially the TDSA approach, which reaches $20 \mathrm{~J}$ with the highest data loads value. Both IEEE 802.15.4 and BARBEI methods present nearly the same 
FIGURE 10 Energy consumed in collision state of the node with fault energy (node number 30)
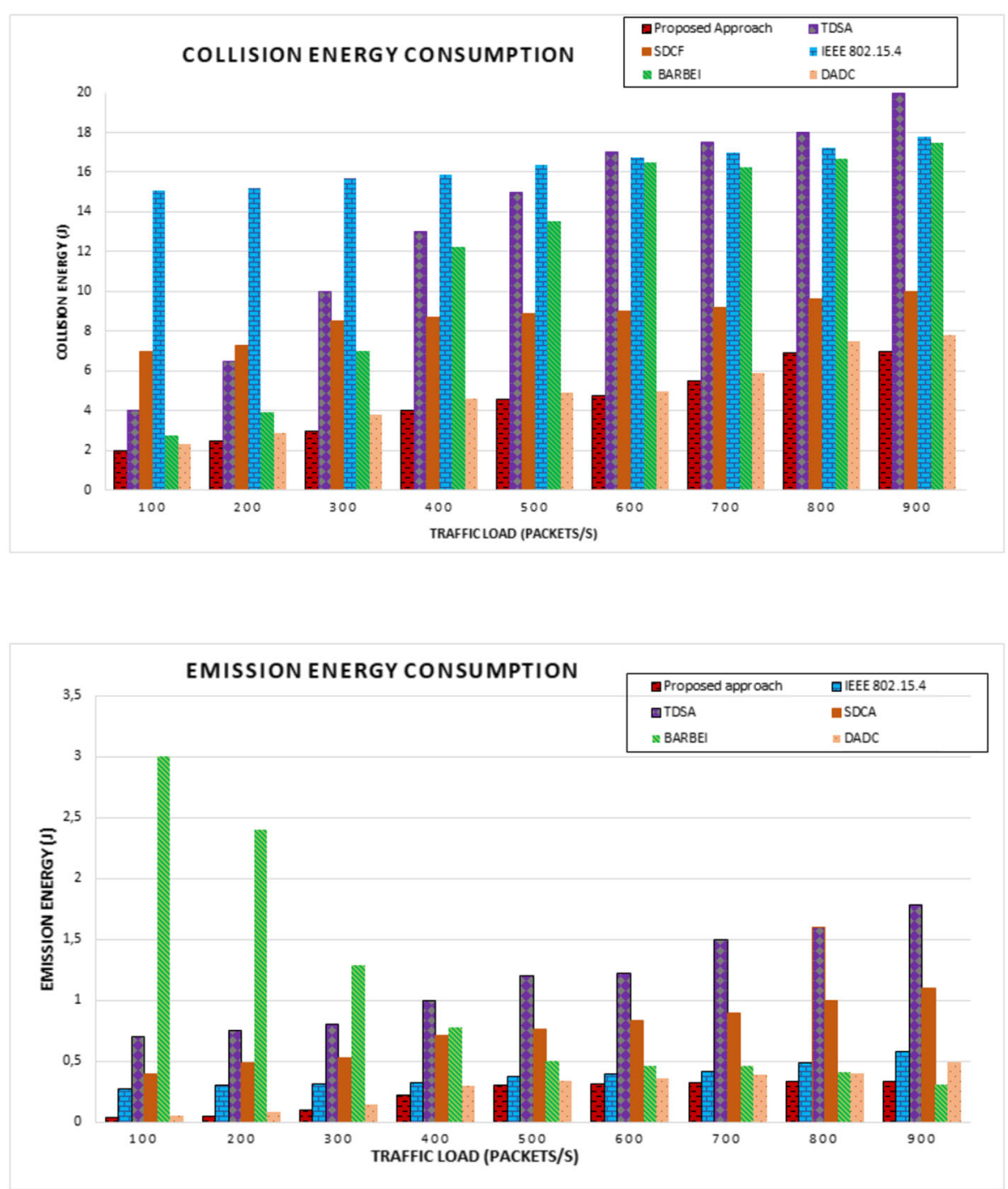

FIGURE 11 Energy consumed in emission state of the node with fault energy (node number 30)

performances (17.9 and $17.5 \mathrm{~J}$, respectively) with high values of the packets loads (more than 600 packets per second). The energy consumed for both DADC and SDCF increases slowly by the data loads, respectively from 7 to $10 \mathrm{~J}$ and from 2 to $7 \mathrm{~J}$, although the simulation results prove the efficiency of the proposed approach, which shows the least energy consumed compared with all other methods (TDSA, DADC, SDCF, BARBEI, and the IEEE 802.15.4).

For the emission state illustrated in Figure 11, the TDSA presents the most important increase rate from 0.7 to $1.7 \mathrm{~J}$ compared with the SDCA approach, which increases from 0.4 to 1.2 J. DADC algorithm achieves interesting results compared with the IEEE 802.15.4 algorithm despite their similar behaviors. Although BARBEI presents an interesting behavior compared with all other methods because of the decrease of its values from 3 to $0.3 \mathrm{~J}$, the proposed approach reaches the best results compared with all the other approaches.

Concerning the overmitting and overhearing state, our approach achieves the best results between 100 and 500 packets per second as presented by Figure 12. Even in the reception state, our technique seems to attain the best results regarding all other approaches in Figure 13. The values reached by the proposed approach do not exceed 1.2 J. On the other hand, the energy consumption for the IEEE 802.15.4, TDSA, and the BARBEI methods reaches the highest values, which are respectively 10, 9.2, and $8.9 \mathrm{~J}$. The DADC algorithm' results increase slowly.

For the sleep state, the energy consumption for the BARBEI method presents the highest results $(9 \mathrm{~J})$. It increases with an important speed. Moreover, the TDSA and the IEEE 802.15.4 present also very important results-the values of energy consumed reach 6 and $4.1 \mathrm{~J}$, respectively. However, the SDCA and DADC methods increase very slowly, which presents an interesting behavior. Once again, in the sleep state, our approach displays an important increase in energy consumed, and it always remains the best (Figure 14). 

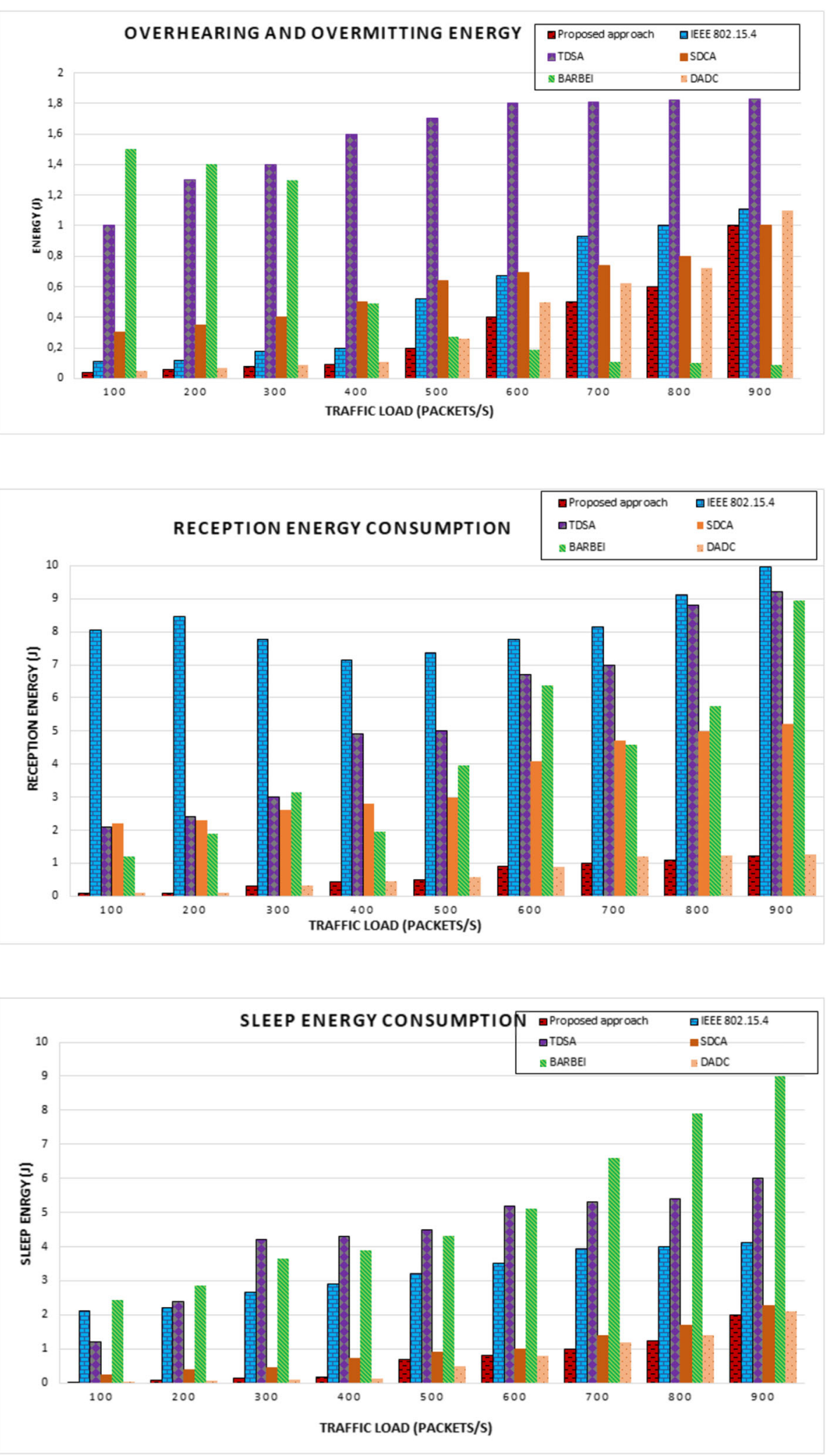

FIGURE 12 Energy consumed in overhearing and overmitting state of the node with fault energy (node number 30)

FIGURE 13 Energy consumed in reception state of the node with fault energy (node number 30)

FIGURE 14 Energy consumed during the sleep state of the node with fault energy (node number 30)

\section{2 | The transient state-related energy consumption}

The idle technique represents the best method to conserve the node's energy. Figure 15 presents the simulation results of the proposed approach and all other techniques: TDSA, DADC, SDCF, BARBEI, and the IEEE 802.15.4. With the increase of the data packets, different behaviors are presented by the different approaches. So, the TDSA, the BARBEI, the IEEE 802.15.4, and our approach decrease. Although the SDCA and the DADC increase, the proposed approach presents the 
FIGURE 15 Energy consumed in idle state of the node with fault energy (node number 30)

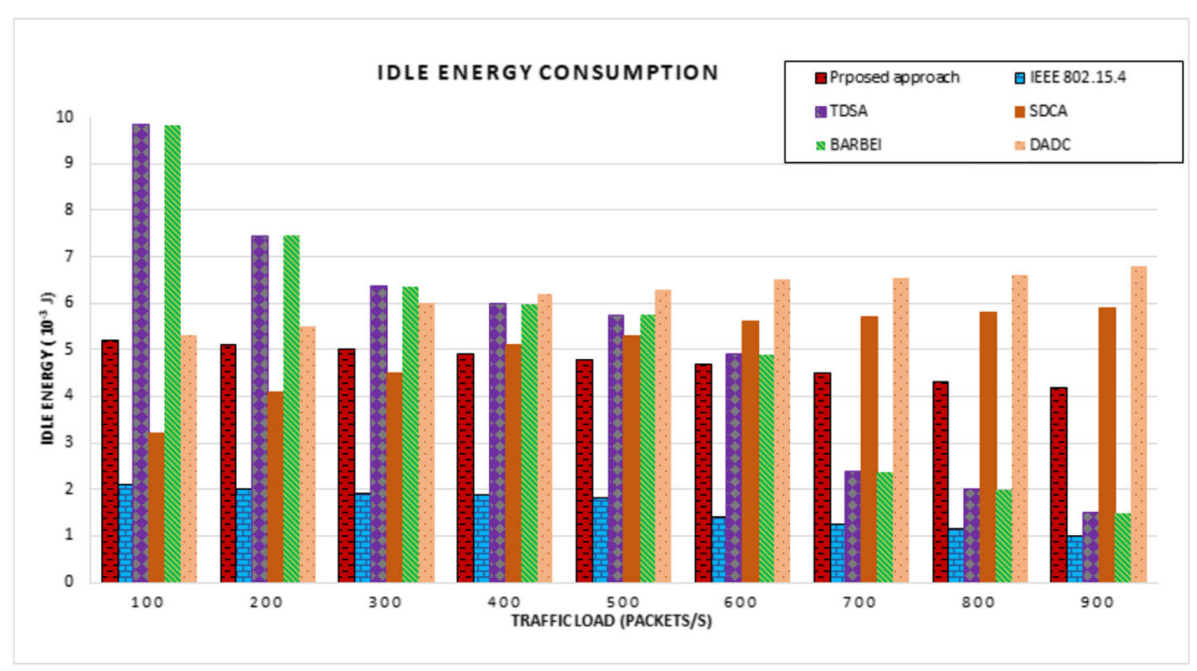

best results compared with the DADC and the SDCA algorithms. As our methodology proves its efficiency in controlling the energy consumption in the WSN, it will be very effective to apply it in such monitoring applications in which the sensor node is inaccessible because of the seriousness of the application environment.

\section{6 | CONCLUSION}

This paper is oriented to establish diverse functional modes in the same network applied in many subgroups. Indeed, every subgroup is characterized by a special IEEE 802.15.4 parameter through its particular duty cycle. Every PAN coordinator sends a beacon frame to its descendants in order to synchronize their activity. In turn, they reply with their current state via their current parameters, which give it the chance to detect their current energy state. In the case where the remaining energy is inferior to the level set, the PAN coordinator intervenes by changing its current IEEE 802.15.4 parameters in order to save its last quantity of energy in the battery. The same process is repeated with all the thresholds. In order to prove the efficiency of the proposed approach, it was compared with five other methods, which are the BARBEI, the DADC, the SDCA, the TDSA, and the IEEE 802.15.4 (with $(\mathrm{BO}, \mathrm{SO})=(7,5)$ ). A validation test bed will be realized in a future work. Moreover, many other network conditions could be also developed such as the QoS. In addition, the efficiency of our approach will be proven with other technologies enabled in the IoT systems such as the IEEE 802.15.6.

\section{ACKNOWLEDGEMENT}

This work was supported by the Ministry of the Higher Education and Scientific Research in Tunisia.

\section{ORCID}

Ahmed Zouinkhi (D) https://orcid.org/0000-0002-1844-975X

Hayfa Ayadi (D) https://orcid.org/0000-0002-9511-6502

\section{REFERENCES}

1. Farooq MU, Waseem M, Mazhar S, Khairi A, Kamal T. A review on Internet of Things (IOT). Int J Comput Appl. 2015;113(1).

2. Malche T, Maheshwary P. Internet of Things (IOT) for building smart home system. In: International Conference on IoT in Social, Mobile, Analytics and Cloud (I-SMAC), 10-11 February, Coimbatore, India; 2017:65-70.

3. Puiu D, Bischof S, Serbanescu B, Nechifor S, Parreira J, Schreiner H. A public transportation journey planner enabled by IoT data analytics. In: 20th Conference on Innovations in Clouds, Internet and Networks (ICIN), March 7-9, 2017, Paris, France:355-359, 2017.

4. Minbo L, Zhu Z, Guangyu C. Information service system of agriculture IOT. automatika. 2013;54(4):415-426.

5. Fernandez F, Pallis GC. Opportunities and challenges of the Internet of Things for healthcare: systems engineering perspective. In: 4th International Conference on Wireless Mobile Communication and Healthcare (Mobihealth), November 3-5, 2014, Athens, Greece; 2014:263-266.

6. Zolotová I, Bundzel M, Lojka T. Industry IoT gateway for cloud connectivity. In: IFIP International Conference on Advances in Production Management Systems, September 7-9, 2015, Tokyo, Japan; 2015:59-66. 
7. Kim NJ, Park JK. Sports analytics \& risk monitoring based on hana platform: sports related big data \& IoT trends by using hana in-memory platform. In: International on SoC Design Conference (ISOCC), November 2-5, Gyeongju, South Korea; 2015:221-222.

8. Alemdar H, Ersoy C. Wireless sensor networks for healthcare: a survey. Comput Netw. 2010;54(15):2688-2710.

9. Mekki K, Derigent W, Zouinkhi A, Rondeau E, Thomas A, Abdelkrim MN. Non-localized and localized data storage in large-scale communicating materials: probabilistic and hop-counter approaches. Comput Stand \& Interfaces. 2016;44:243-257.

10. Saihi M, Zouinkhi A, Boussaid B, Abdelkarim MN, Andrieux G. Hidden Gaussian Markov model for distributed fault detection in wireless sensor networks. Trans Inst Meas Control. 2018;40(6):1788-1798.

11. Ayadi H, Zouinkhi A, Boussaid B, Abdelkrim MN, Val Thierry. Energy efficiency in WSN: IEEE 802.15.4. In: IEEE International Conference on Sciences and Techniques of Automatic Control and Computer Engineering (STA), December 19-21, 2016 Sousse, Tunisia; 2016:766-771.

12. Ayadi H, Zouinkhi A, Boussaid B, Abdelkrim MN, Val T, Khssibi S. An effective approach for energy aware in wireless sensor network. In: IEEE International Conference on Smart, Monitored and Controlled Cities (SM2C), Feb 17-19, Sfax, Tunisa; 2017:128-133.

13. Zhang D-g, Song X-d, Wang X, Ma Y-y. Extended AODV routing method based on distributed minimum transmission (DMT) for WSN. AEU-Int J Electron C. 2015;69(1):371-381.

14. Zhang D-g, Liu S, Liu X-h, Zhang T, Cui Y-y. Novel dynamic source routing protocol (DSR) based on genetic algorithm-bacterial foraging optimization (GA-BFO). Int J Commun Syst. 2018;31(18).

15. Zhang D-G, Zheng K, Zhang T, Wang X. A novel multicast routing method with minimum transmission for WSN of cloud computing service. Soft Comput. 2015;19(7):1817-1827.

16. Zhang D-g, Wang X, Song X-d, Zhang T, Zhu Y-n. A new clustering routing method based on PECE for WSN. EURASIP J Wirel Commun Netw. 2015;2015(162).

17. Zhang D-g, Zhang T, Dong Y, Liu X-h, Cui Y-y, Zhao D-x. Novel optimized link state routing protocol based on quantum genetic strategy for mobile learning. J Netw Comput Appl. 2018;122:37-49.

18. Zhang D-g, Liu S, Zhang T, Liang Z. Novel unequal clustering routing protocol considering energy balancing based on network partition \& distance for mobile education. J Netw Comput Appl. 2017;88:1-9.

19. Zhang D-g, Niu H-1, Liu S. Novel PEECR-based clustering routing approach. Soft Comput. 2017;21(24):7313-7323.

20. Zhang D-g, Wang X, Song X-d. New medical image fusion approach with coding based on SCD in wireless sensor network. $J$ Electr Eng Technol. 2015;10(6):2384-2392.

21. Zhang D-g, Li W-b, Liu S, Zhang X-d. Novel fusion computing method for bio-medical image of WSN based on spherical coordinate. $J$ Vibroengineering. 2016;18(1).

22. Zhang D, Wang X, Song X, Zhao D. A novel approach to mapped correlation of ID for RFID anti-collision. IEEE Trans Serv Comput. 2014;7(4):741-748.

23. Zhang D-g, Chen C, Cui Y-y, Zhang Ting. New method of energy efficient subcarrier allocation based on evolutionary game theory. Mob Netw Appl. 2018:1-14.

24. Zhang D-G. A new approach and system for attentive mobile learning based on seamless migration. Appl Intell. 2012;36(1):75-89.

25. Zhang D-g, Zheng K, Zhao D-X, Song X-d, Wang X. Novel quick start (QS) method for optimization of TCP. Wirel Netw. 2016;22(1):211-222.

26. Chen J, Mao G, Li C, Liang W, Zhang D-g. Capacity of cooperative vehicular networks with infrastructure support: multiuser case. IEEE Trans Veh Technol. 2018;67(2):1546-1560.

27. Zhang D, Ge H, Zhang T, Cui Y-Y, Liu X, Mao G. New multi-hop clustering algorithm for vehicular ad hoc networks. IEEE Trans Intell Transp Syst. 2019;20(4):1517-1530.

28. Ma Z, Zhang D-g, Chen J, Hou Y-x. Shadow detection of moving objects based on multisource information in Internet of Things. $J$ Exp Theor Artif Intell. 2017;29(3):649-661.

29. Zhang D-g, Zhou S, Chen J, Liu S. New mixed adaptive detection algorithm for moving target with big data. J Vibroengineering. 2016;18(7).

30. Zhang D-g, Liang Y-p. A kind of novel method of service-aware computing for uncertain mobile applications. Math Comput Modell. 2013;57(3-4):344-356.

31. Zhang D, Kang X, Wang J. A novel image de-noising method based on spherical coordinates system. EURASIP Journal on Advances in Signal Processing. 2012;2012(110).

32. Zhang D, Zhao C-p, Liang Y-p, Liu Z-j. A new medium access control protocol based on perceived data reliability and spatial correlation in wireless sensor network. Comput Electr Eng. 2012;38(3):694-702.

33. Zhang D-G, Zhu Y-N, Zhao C-P, Dai W-B. A new constructing approach for a weighted topology of wireless sensor networks based on local-world theory for the Internet of Things (IOT). Comput Math Appl. 2012;64(5):1044-1055.

34. Zhang D-g, Niu H-l, Liu S, Ming X-c. Novel positioning service computing method for WSN. Wirel Pers Commun. 2017;92(4):1747-1769.

35. Zhang D-g, Song X-d, Wang X, Li K, Li W-b, Ma Z. New agent-based proactive migration method and system for big data environment (BDE). Eng Comput. 2015;32(8):2443-2466.

36. Ma Z, Zhang D, Liu S, Song J, Hou Y. A novel compressive sensing method based on SVD sparse random measurement matrix in wireless sensor network. Eng Comput. 2016;33(8):2448-2462.

37. Zhang D-g, Zhang T, Zhang J, Dong Y, Zhang X-d. A kind of effective data aggregating method based on compressive sensing for wireless sensor network. EURASIP J Wirel Commun Netw. 2018;2018(159).

38. Liu S, Zhang D-G, Liu X-h, Zhang T, Gao J-x, Cui Y-y, et al. Dynamic analysis for the average shortest path length of mobile ad hoc networks under random failure scenarios. IEEE Access. 2019;7:21343-21358.

39. Zhang D, Gao J-x, Liu X-h, Zhang T, Zhao D-x. Novel approach of distributed adaptive trust metrics for MANET. Wirel Netw. 2019:1-17. 
40. Zhang D, Zhang T, Liu X. Novel self-adaptive routing service algorithm for application in VANET. Appl Intell. 2019;45(5):1866-1879.

41. Zhang D-g, Tang Y-m, Cui Y-y, Gao J-x, Liu X-h, Zhang T. Novel reliable routing method for engineering of internet of vehicles based on graph theory. Eng Comput. 2018;36(1):226-247.

42. Zhang D-g, Li G, Zheng K, Ming X, Pan Z-H. An energy-balanced routing method based on forward-aware factor for wireless sensor networks. IEEE Trans Ind Inf. 2014;10(1):766-773.

43. Zhang D-g, Zhang X-d. Design and implementation of embedded un-interruptible power supply system (EUPSS) for web-based mobile application. Int J Enterp Inf Syst. 2012;6(4):473-489.

44. Zhang D-g, Zhou S, Tang Y-m. A low duty cycle efficient MAC protocol based on self-adaption and predictive strategy. Mob Netw Appl. 2018;23(4):828-839.

45. Lee B-H, Wu H-K. Study on a dynamic superframe adjustment algorithm for IEEE 802.15.4 LR-WPAN. In: IEEE Vehicular Technology Conference (VTC 2010), 16-19 May 2010, Taipei, Taiwan; 2010:1-5.

46. Choudhury N, Matam R, Mukherjee M, Shu Lei. Dynamic adaptation of duty cycling with MAC parameters in cluster tree IEEE 802.15.4 networks. In: Annual conference of the IEEE Industrial Electronics Society, 29 October-1 November, Bejing, China; 2017:3449-3454.

47. Barbieri A, Chiti F, Fantacci R. WSN17-2: proposal of an adaptive MAC protocol for efficient IEEE 802.15.4 low power communications. In: Global Telecommunications Conference, (GLOBECOM'06), November 27- December 1, San Fracisco, California, USA; 2006:1-5.

48. Latif U, Farhad A, Zafar NA. A smart duty cycle adaptation algorithm for the low rate wireless personal area networks. In: 19th International Multi-Topic Conference (INMIC), December 5-6, Islamabad, Pakistan; 2016:1-6.

49. Farhad A, Zia Y, Farid S, Hussain FB. A traffic aware dynamic super-frame adaptation algorithm for the IEEE 802.15 .4 based networks. In: IEEE Asia Pacific Conference On Wireless And Mobile (APWiMob), August 27-29, Bandung, Indonesia; 2015:261-266.

50. Gao B, He C. An individual beacon order adaptation algorithm for IEEE 802.15.4 networks. In: 11th IEEE Singapore International Conference On Communication Systems, November 19-21, Guangzhou, China; 2008:12-16.

51. Oliveira CHS, Ghamri-Doudane Y, Lohier S. A duty cycle self-adaptation algorithm for the 802.15.4 wireless sensor networks. In: Global Information Infrastructure Symposium, October 28-31, Trento, Italy; 2013:1-7.

52. Tan J, Koo SGM. A survey of technologies in Internet of Things. In: IEEE International Conference on Distributed Computing in Sensor Systems (DCOSS), May 26-28, Marina Del Rey, California; 2014:269-274.

53. Society IC. IEEE 802.15.4 wireless LAN medium access control MAC and physical layer (phy) specifications for low-rate wireless personal area networks LR-WPANS; 2006.

54. Ha JY, Kim TH, Park HS, Choi S, Kwon WH. An enhanced CSMA/CA algorithm for IEEE 802.15.4 LR-WPANS. IEEE Commun Lett. 2007;11(5).

55. Varga Andras. Omnet++. In: Modeling and tools for network simulation; 2010:35-59.

56. Salayma M, Al-Dubai A, Romdhani I, Yassein MB. Battery aware beacon enabled IEEE 802.15.4: an adaptive and cross-layer approach. In: Federated Conference on Computer Science and Information Systems (FedCSIS), 13-16 September, Lodz, Poland; $2015: 1267-1272$.

57. Kavian YS, Rasouli H. Adaptive IEEE 802.15.4 MAC protocol for wireless sensor networks. In: Technological breakthroughs in modern wireless sensor applications. IGI Global; 2015:109-123. 